\title{
Genetically Selected Cardiomyocytes from Differentiating Embryonic Stem Cells Form Stable Intracardiac Grafts
}

\author{
Michael G. Klug, Mark H. Soonpaa, Gou Young Koh, and Loren J. Field \\ Krannert Institute of Cardiology, Indiana University School of Medicine, Indianapolis, Indiana 46202-4800
}

\begin{abstract}
This study describes a simple approach to generate relatively pure cultures of cardiomyocytes from differentiating murine embryonic stem (ES) cells. A fusion gene consisting of the $\alpha$-cardiac myosin heavy chain promoter and a cDNA encoding aminoglycoside phosphotransferase was stably transfected into pluripotent ES cells. The resulting cell lines were differentiated in vitro and subjected to G418 selection. Immunocytological and ultrastructural analyses demonstrated that the selected cardiomyocyte cultures $(>99 \%$ pure) were highly differentiated. G418 selected cardiomyocytes were tested for their ability to form grafts in the hearts of adult dystrophic mice. The fate of the engrafted cells was monitored by antidystrophin immunohistology, as well as by PCR analysis with primers specific for the myosin heavy chain-aminoglycoside phosphotransferase transgene. Both analyses revealed the presence of ES-derived cardiomyocyte grafts for as long as $7 \mathrm{wk}$ after implantation, the latest time point analyzed. These studies indicate that a simple genetic manipulation can be used to select essentially pure cultures of cardiomyocytes from differentiating ES cells. Moreover, the resulting cardiomyocytes are suitable for the formation of intracardiac grafts. This selection approach should be applicable to all ES-derived cell lineages. (J. Clin. Invest. 1996. 98:216-224.) Key words: myocardial repair • infarct • somatic cell therapy
\end{abstract}

\section{Introduction}

Cardiomyocyte loss in the adult mammalian heart is irreversible and frequently leads to diminished cardiac function. The ability to increase the number of functional cardiomyocytes in a diseased heart would have obvious therapeutic potential. Recent studies have suggested that cellular engraftment can be used to augment myocyte number in the adult heart. Although several different types of myocyte preparations have been successfully engrafted (1-8), the inherent electrophysiologic, structural, and contractile properties of cardiomyocytes strongly suggest that they are the ideal donor cell type. However, difficulties in obtaining sufficient numbers of fetal donor cells might con-

Address correspondence to Loren J. Field, Krannert Institute of Cardiology, 1111 West 10th Street, Indianapolis, IN 46202-4800. Phone: 317-630-7776; FAX: 317-274-9697. Gou Young Koh's present address is Department of Physiology, Chonbuk Medical School, San 2-20, Keum-Am-Dong, Chonju 560-182, South Korea.

Received for publication 4 December 1995 and accepted in revised form 12 April 1996.

J. Clin. Invest.

(C) The American Society for Clinical Investigation, Inc.

0021-9738/96/07/216/09 \$2.00

Volume 98, Number 1, July 1996, 216-224 stitute a serious impediment for the widespread implementation of intracardiac engraftment. Identification of an alternative source of donor cardiomyocytes would thus be of value.

Embryonic stem $(\mathrm{ES})^{1}$ cells, totipotent cell lines derived from the inner cell mass of blastocysts, have the ability to differentiate into a variety of cell lineages in vitro (9). Cardiogenic induction during ES differentiation is manifest by the appearance of spontaneously and rhythmically contracting myocytes. ES-derived cardiomyocytes express $\alpha$ - and $\beta$-cardiac myosin heavy chain (MHC) (10), $\alpha$-tropomyosin (11), myosin light chain $2 \mathrm{v}$ (MLC-2v) and atrial natriuretic factor (12), phospholamban (13) and type B natriuretic factor (14), and exhibit normal contractile sensitivity to calcium (15). Electrophysiologic analyses have identified action potentials typical for atrial, ventricular, and conduction system cardiomyocytes from ES-isolated cell preparations $(16,17)$. Furthermore, cell cycle withdrawal and multinucleation in ES-derived cardiomyocytes follow a temporal program roughly similar to that observed during cardiogenesis in vivo (18). These attributes suggested that differentiating ES cells might constitute a renewable source of donor cardiomyocytes suitable for cardiac engraftment.

However, successful use of ES-derived donor cells would require the generation of essentially pure cardiomyocyte cultures, as engraftment of pluripotent or totipotent ES cells would likely result in teratoma formation. In this study, totipotent ES cells were genetically modified so as to permit cardiomyocyte enrichment. ES cell lines carrying a fusion gene comprised of the $\alpha$-cardiac MHC promoter and sequences encoding aminoglycoside phosphotransferase were generated. Expression of the fusion gene in ES-derived cardiomyocytes facilitated their selection with G418 after in vitro differentiation. Immunohistologic and ultrastructural analyses revealed that the selected cardiomyocytes were highly differentiated. The G418 selected cardiomyocytes were tested for their ability to form grafts in the hearts of adult mice. Stable ES-derived cardiomyocyte grafts were observed as long as 7 wk after implantation. Thus, a relatively simple scheme of genetic selection can be used to generate pure cultures of differentiated cardiomyocytes. Moreover, the selected cells were able to form stable intracardiac grafts. This selection approach should be applicable to all ES-derived cell lineages.

\section{Methods}

Generation of the myosin heavy chain-aminoglycoside phosphotransferase transgene. A molecule carrying both an $\alpha$-cardiac myosin heavy chain-aminoglycoside phosphotransferase (MHC-neor) and a phosphoglycerate kinase (pGK)-hygromycin resistant transgene in a common pBM20 vector backbone (Boehringer Mannheim, Indianapolis,

1. Abbreviations used in this paper: ES, embryonic stem; LIF, leukemia inhibitory factor; MHC, myosin heavy chain; MLC, myosin light chain; neo ${ }^{\mathrm{r}}$, neomycin resistance; pGK, phosphoglycerate kinase. 
IN) was generated. The $\alpha$-cardiac MHC promoter consisted of $4.5 \mathrm{~kb}$ of $5^{\prime}$ flanking sequence and $1 \mathrm{~kb}$ of the gene encompassing exons 1 through 3 up to but not including the initiation codon (19). The aminoglycoside phosphotransferase $\left(\right.$ neo $\left.^{\mathrm{r}}\right) \mathrm{cDNA}$ was subcloned from pMC1-neo poly A (Stratagene, La Jolla, CA). The pGK-hygromycin sequences were described previously (20). The transgene is depicted schematically in Fig. 1. Transgene insert (containing both the MHC-neo ${ }^{r}$ and pGK-hygromycin sequences) was isolated by digestion with XhoI and HinDIII and transfected into ES-D3 stem cells (American Type Culture Collection, Rockville, MD) via electroporation. Transfected clones were selected by growth in the presence of hygromycin $(200 \mu \mathrm{g} / \mathrm{ml}$; Calbiochem-Novabiochem, La Jolla, CA). PCR analyses were used to confirm that both the MHC-neo ${ }^{\mathrm{r}}$ and pGK-hygromycin sequences were present in the resulting cell lines. The ES cells were maintained in the undifferentiated state by culturing in high glucose DME containing $10 \%$ FBS, nonessential amino acids, and $0.1 \mathrm{mM}$ 2-mercaptoethanol. The medium was supplemented to a final concentration of $100 \mathrm{U} / \mathrm{ml}$ with conditioned medium containing recombinant leukemia inhibitory factor (LIF) as described (21).

In vitro differentiation and selection of ES-derived cardiomyocytes. To induce differentiation, $2 \times 10^{6}$ freshly dissociated ES cells were plated onto a $100-\mathrm{mm}$ bacterial Petri dish containing $10 \mathrm{ml}$ of DME lacking supplemental LIF. After $3 \mathrm{~d}$ in suspension culture, the resulting embryoid bodies were plated onto plastic 100-mm cell culture dishes and allowed to attach. Regions of cardiogenesis were readily identified by the presence of spontaneous contractile activity. For cardiomyocyte selection, the differentiated cultures were grown for $8 \mathrm{~d}$ in the presence of G418 $(200 \mu \mathrm{g} / \mathrm{ml}$; Gibco Laboratories, Grand Island, NY).

To determine the cardiomyocyte content in nonselected cultures, plates were treated with trypsin $16 \mathrm{~d}$ after cardiogenic induction, and the resulting suspension was plated at low density onto fibronectincoated cell culture dishes to permit visualization of individual cells. After $24 \mathrm{~h}$ of culture, the cells were fixed in acetone and processed for immunocytology with MF20, an anti-MHC antibody (see below). To determine the cardiomyocyte content following physical enrichment protocols, rhythmically contracting regions were microdissected with a sterile Pasteur pipette $16 \mathrm{~d}$ after cardiogenic induction, dissociated with trypsin, and processed for MHC immunoreactivity. To determine the cardiomyocyte content in selected cultures, ES cells were allowed to differentiate for $8 \mathrm{~d}$ following cardiogenic induction. The cultures were then subjected to G418 selection for an additional $8 \mathrm{~d}$. The selected cells were dissociated and processed for MHC immunoreactivity.

Immunofluorescence analyses of cultured ES-derived cardiomyocytes. Cultures of selected EA3 cardiomyocytes were treated with trypsin, and the resulting suspension was cultured at low density onto fibronectin-coated cell culture dishes for $24 \mathrm{~h}$ to permit visualization of individual cells. The cultures were then fixed in acetone, and nonspecific immunoreactivity was blocked by incubation in $1 \%$ BSA, $10 \%$ goat serum, $1 \times$ PBS. The primary antibodies used were: MF20 (antisarcomeric myosin), 9D10 (antititin), EA-53 (anti- $\alpha$-actinin; Sigma Immunochemicals, St. Louis, MO), D3 (antidesmin), and 6-10 (antidystrophin). Secondary antibody was fluorescein- or rhodamine-

\section{MHC-neo'}

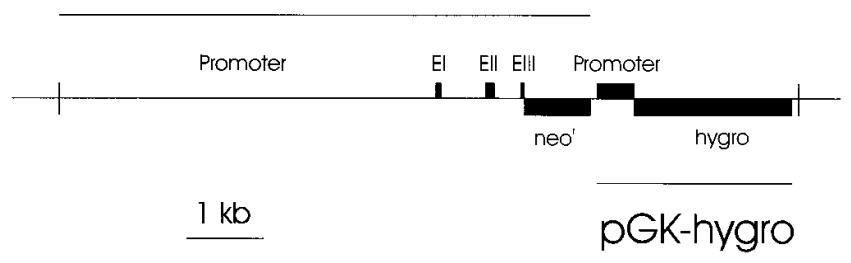

Figure 1. Structure of the MHC-neo ${ }^{\mathrm{r}} / \mathrm{pGK}$-hygromycin transgene. The plasmid backbone was pBM20. conjugated anti-mouse or anti-rabbit $\operatorname{IgG~} \mathrm{F}\left(\mathrm{ab}^{\prime}\right)_{2}$ fragment (Boehringer Mannheim). All samples were counterstained with Hoechst 33342 (Molecular Probes, Inc., Eugene, OR) and visualized by epifluorescence.

Ultrastructural analysis of selected ES-derived cardiomyocytes. Selected cell cultures were fixed in $2 \%$ glutaraldehyde in $0.1 \mathrm{M}$ sodium cacodylate buffer ( $\mathrm{pH} 7.4$ ) containing $0.1 \mathrm{M}$ sucrose and $0.05 \%$ $\mathrm{caCl}_{2}$, followed by postfixation in $2 \%$ osmium tetroxide in the same buffer (Stevens Metallurgical Corp., New York). All other chemicals were obtained from Ladd Research Industries, Inc. (Burlington, VT). Samples were stained with $2 \%$ uranyl acetate in $\mathrm{pH} 5.2$ maleate buffer $(0.05 \mathrm{M})$, dehydrated, and embedded in Ladd LX-112. After trimming, the block was thin sectioned and stained with uranyl acetate and lead citrate. Specimens were viewed on a Phillips 400 transmission electron microscope.

Myocardial engraftment of selected ES-derived cardiomyocytes. Cultures of selected ES-derived cardiomyocytes were digested with trypsin, and the resulting single cell preparation was washed three times with $1 \times$ DME and directly injected into the ventricular myocardium of anesthetized, intubated adult mdx mice (The Jackson Laboratory, Bar Harbor, ME) using a 30-gauge tuberculin syringe as described previously (8). After extubation and evacuation of the pneumothorax, the animals were placed at $37^{\circ} \mathrm{C}$ and monitored until they recovered from surgery. All animal procedures were in accordance with institutional and National Institutes of Health guidelines.

Immunohistology of hearts. Hearts were harvested, cryoprotected in $30 \%$ sucrose, embedded and sectioned at $10 \mu \mathrm{m}$ on a cryomicrotome as described previously $(7,8)$. These studies used fetal mice (embryonic day $15 \mathrm{C} 3 \mathrm{HeB} / \mathrm{FeJ})$, normal adult mice $(\mathrm{C} 3 \mathrm{HeB} / \mathrm{FeJ})$, and dystrophic adult mice $(\mathrm{C} 57 \mathrm{Bl} / 10 \mathrm{ScSn}-\mathrm{mdx} / \mathrm{J})$. All animals were obtained from The Jackson Laboratory. Nonspecific immunoreactivity was blocked by incubation in $1 \%$ BSA, $10 \%$ goat serum, $1 \times$ PBS. For antidystrophin immunohistology, sections were incubated with 6-10 (gift of Dr. T. Byers, Indiana University) or DYS1 (Novocastra Laboratories, Newcastle-upon-Tyne, United Kingdom) in $1 \times$ PBS, $1 \%$ BSA for $2 \mathrm{~h}$ at $25^{\circ} \mathrm{C}$, rinsed and reacted with a fluorescein-conjugated secondary antibody.

Northern blot analyses. Tissue or cells were homogenized in 4.0 $\mathrm{M}$ guanidinium thiocyanate and RNA purified by centrifugation through 5.7 M CsCl using standard protocols as described (22). RNA samples were quantitated by spectrophotometry at $260 \mathrm{~nm}$. For Northern analysis, RNA was denatured with glyoxal, separated by size on $1.2 \%$ agarose gels, and transferred to Genescreen (DuPont, Wilmington, DE). Oligonucleotide probes were labeled using polynucleotide kinase under standard conditions (22). Hybridizations were for $20 \mathrm{~h}$ at $65^{\circ} \mathrm{C}$ in $4 \times$ SSC, $2 \times$ Denhardt's, $0.1 \%$ SDS, and 1 $\mathrm{mg} / \mathrm{ml}$ salmon sperm DNA. Blots were washed at $60^{\circ} \mathrm{C}$ in $2 \times \mathrm{SSC}$, $0.1 \%$ SDS, and signal was visualized by autoradiography at $-70^{\circ} \mathrm{C}$ with an intensifying screen. The MLC-2v probe was 5'-CACAGCCCTGGGATGGAGAGTGGGCTGTGGGTCACCTGAGGCTGTGGTTCAG-3'; the MLC-2a probe was 5'-GAGGTGACCTCAGCCTGTCTACTCCTCTTTCTCATCCCCG-3'. Quality of the RNA samples was confirmed by Northern analysis with a murine 18s rRNA oligonucleotide probe (5'-TCCATTATTCCTAGTGCGGTATCCAGGAGGATCGGGCCTGCTTT-3').

Southern blot analyses. DNA from EA3 cells or from sections of engrafted hearts was prepared by digestion in a solution containing proteinase $\mathrm{K}(0.1 \mu \mathrm{g} / \mathrm{ml}), 50 \mathrm{mM} \mathrm{KCl}, 10 \mathrm{mM}$ Tris- $\mathrm{HCl}, \mathrm{pH} 8.3,2.5$ $\mathrm{mM} \mathrm{MgCl}_{2}, 10 \mathrm{mM}$ DTT, $0.005 \%$ SDS. The samples were digested at $55^{\circ} \mathrm{C}$ for $2-4 \mathrm{~h}$. The proteinase $\mathrm{K}$ was heat inactivated and the resulting crude nucleic acid preparation was amplified by PCR as described previously (23). The sense primer (5'-CTCTGACAGAGAAGCAGGCACTTTACATGG- $3^{\prime}$ ) was located in the $\alpha$-MHC promoter and the antisense primer ( $5^{\prime}$-ATAGCCGCGCTGCCTCGTCCTGCAGTTCATTCA-3') was located in the neo ${ }^{r}$ cDNA. The amplification products were transferred to Genescreen Plus (DuPont) and hybridized with a nick-translated probe using standard conditions (22). The hybridization probe encompassed sequences of the MHC-neo ${ }^{\mathrm{r}}$ trans- 
gene internal to the oligonucleotide primers used for PCR amplification.

\section{Results}

The structure of the MHC-neo $/$ pGK-hygromycin transgene is depicted in Fig. 1. Initial transfection experiments produced five hygromycin-resistant ES cell lines. PCR analyses confirmed that the cell lines carried both the MHC-neor and the pGK-hygromycin fusion genes (Klug, M.G., unpublished observation). One line, designated EA3, was chosen for subsequent analyses. To induce differentiation, EA3 cells were plated in suspension culture in the absence of supplementary LIF. 3 d later, the resulting embryoid bodies were replated
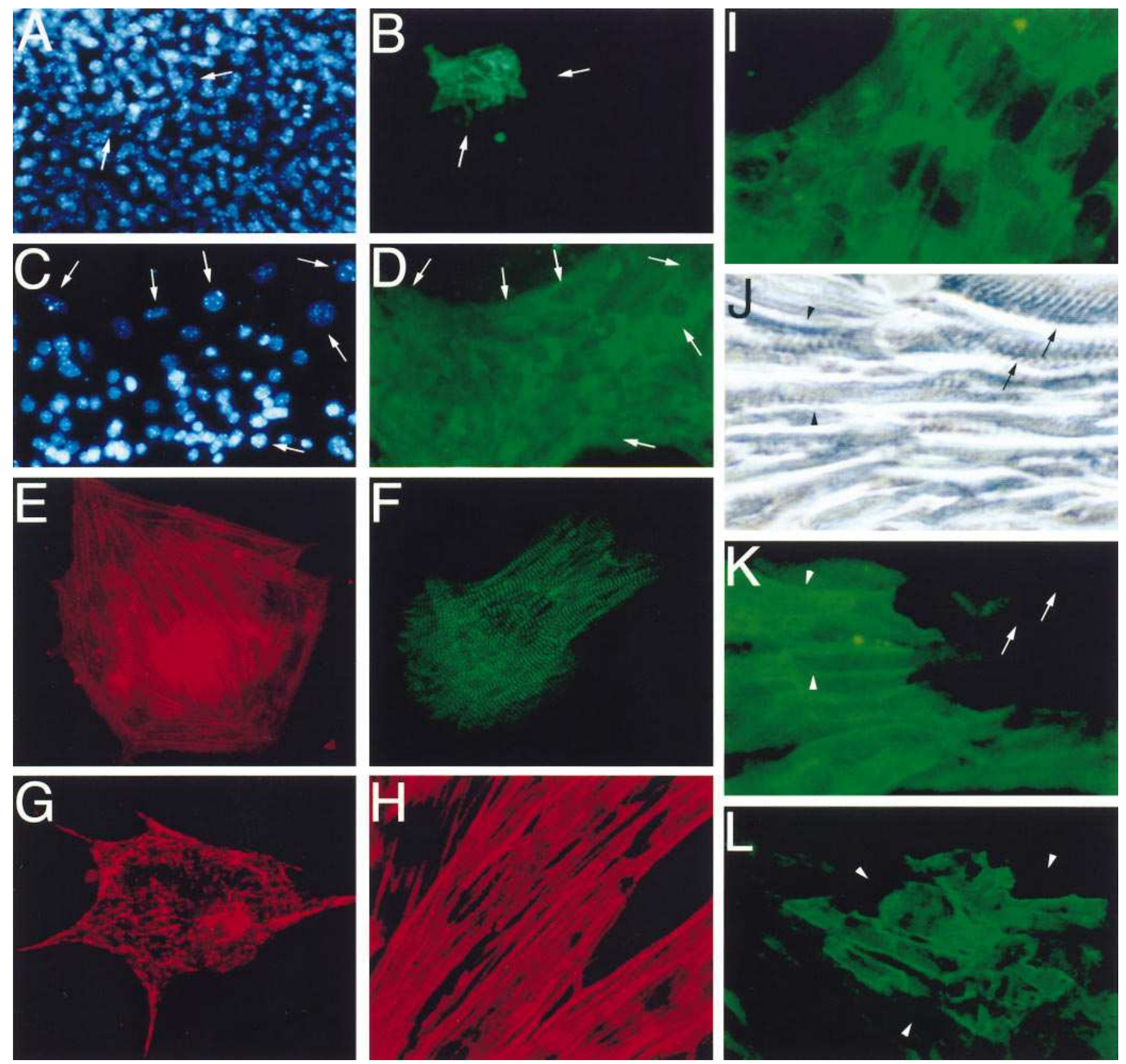

Figure 2. G418 selection of ES-derived cardiomyocytes in vitro and formation of intracardiac grafts. $(A)$ Hoechst epifluorescence of a nonselected culture of EA3 ES cells $16 \mathrm{~d}$ after differentiation was induced. Note the high density of cells present in the field. $(B)$ Antisarcomeric MHC immunofluorescence (green signal) of the same field depicted in $A$. Note that only a few cells are cardiomyocytes (arrows demarcate the same group of cells in $A$ and $B$ ). (C) Hoechst epifluorescence of a G418-selected culture of EA3 ES cells $16 \mathrm{~d}$ after cardiogenic induction. $(D)$ Antisarcomeric myosin immunofluorescence (green signal) of the same field depicted in $C$. Note that all of the cells presented in the G418-selected culture express sarcomeric myosin (arrows demarcate the same group of cells in $C$ and $D)$. $(E-I)$ Immunocytologic analysis for titin, $\alpha$-actinin, desmin, sarcomeric myosin, and dystrophin expression, respectively, in G418-selected EA3 cardiomyocytes. $(J$ and $K$ ) Phase-contrast image and antidystrophin immunofluorescence, respectively, of an $\mathrm{mdx}$ heart engrafted with G418-selected cardiomyocytes. The same field is depicted in $J$ and $K$, and dystrophin immunoreactivity appears as green signal in $K$. Arrows indicate myofiber-containing host cells (dystrophin negative), while arrowheads indicate myofiber-containing donor cells (dystrophin positive). ( $L$ ) Antidystrophin immunofluorescence of a second G418selected cardiomyocyte graft. Dystrophin immunoreactivity appears as green signal. 
Table I. Relative Cardiomyocyte Content in Nonselected, Physically Selected, and G418 Selected Cultures of Differentiating EA3 ES Cells

\begin{tabular}{lccc}
\hline \multicolumn{1}{c}{ Preparation } & $\begin{array}{c}\text { Sarcomeric myosin } \\
\text { positive cells }\end{array}$ & $\begin{array}{c}\text { Sarcomeric myosin } \\
\text { negative cells }\end{array}$ & $\begin{array}{c}\text { Percent } \\
\text { cardiomyocytes }\end{array}$ \\
\hline No selection* & 11 & 2000 & 0.55 \\
Physical isolation $^{\ddagger}$ & 68 & 2000 & 3.4 \\
G418 selection $^{\S}$ & 791 & 3 & 99.6 \\
\hline
\end{tabular}

Analyses were performed on multiple dishes obtained from the same differentiating culture to control for variation in cardiogenic induction.

* $16 \mathrm{~d}$ after cardiogenic induction, plates were treated with trypsin and the resulting suspension was plated at low density onto fibronectincoated cell culture dishes. After $24 \mathrm{~h}$ of culture, sarcomeric myosin immunoreactivity was scored (see Methods). ${ }^{\ddagger} 16 \mathrm{~d}$ after cardiogenic induction, rhythmically contracting regions were microdissected with a sterile Pasteur pipette, dissociated with trypsin, and the resulting suspension was plated at low density onto fibronectin-coated cell culture dishes. After $24 \mathrm{~h}$ of culture, sarcomeric myosin immunoreactivity was scored. ${ }^{\S} 8 \mathrm{~d}$ after cardiogenic induction, cultures were treated with G418. After culturing in the presence of G418 for a total of 8 additional d, the selected cells were dissociated with trypsin and the resulting suspension was plated at low density onto fibronectin-coated cell culture dishes. After $24 \mathrm{~h}$ of culture, sarcomeric myosin immunoreactivity was scored.

onto tissue cultures dishes and allowed to form adherent colonies. Hoechst epifluorescence analyses demonstrated a very high cellular density in these cultures after a total of $16 \mathrm{~d}$ of in vitro differentiation. (Fig. $2 \mathrm{~A}$ ). Immunofluorescence analysis of the same field revealed that only a small percentage of the differentiated cells expressed sarcomeric myosin, indicating that the vast majority of the cells were not cardiomyocytes (Fig. 2 B). To quantitate the cardiomyocyte content, the differentiated cultures were digested with trypsin and replated at a lower density to assess the immunoreactivity of individual cells. These analyses confirmed that cardiomyocytes (i.e., MF20 immunoreactive cells) comprised $<1 \%$ of the cultured cells (Table I).

To determine the extent of cardiomyocyte enrichment obtained by simple physical isolation, EA3 cells were differentiated in vitro for $16 \mathrm{~d}$ using the same procedure described above. Regions exhibiting spontaneous contractile activity were microdissected, digested with trypsin, and replated at a density suitable for immunocytologic analysis of individual cells. Although an $\sim 10$-fold enrichment of cardiomyocytes was observed, cardiomyocytes comprised only $3.4 \%$ of the cells present in microdissected cultures (Table I). To determine the extent of cardiomyocyte enrichment obtained with genetic selection, EA3 cells were differentiated using the same approach described above. After 8 d of differentiation, G418 selection was imposed and the cells were cultured for an additional $8 \mathrm{~d}$. Hoechst epifluorescence analyses indicated that the cellular density in these cultures was markedly reduced as compared with similarly aged nonselected EA3 cultures (Fig. 2, $C$ and $A$, respectively). Immunofluorescence analysis of the same field of G418-selected cells indicated that the vast majority of the surviving cells exhibited sarcomeric myosin immunoreactivity (Fig. 2 D). Immunofluorescence analysis of single cell preparations was once again used to quantitate the extent of cardiomyocyte enrichment. More than $99 \%$ of the cells in
G418-selected cultures expressed sarcomeric myosin (Table I). Thus, in the absence of enrichment, only a few cells in differentiated cultures were cardiomyocytes. In contrast, the G418 selection of differentiating EA3 cells produced essentially pure cultures of cardiomyocytes.

Additional studies were performed to characterize the G418-selected cells. Previous studies have shown that the presence of sarcomeric myosin immunoreactivity coupled with the absence of nebulin immunoreactivity permitted the definitive identification of cardiomyocytes in differentiating ES cultures (18). As expected, the G418-selected cells exhibited sarcomeric myosin immunoreactivity but not nebulin immunoreactivity, confirming that the selected cultures were comprised of cardiomyocytes (Table II). The extent of differentiation in G418selected cardiomyocyte cultures was further assessed by immunocytologic analyses. Once again, the selected cultures were digested with trypsin and replated at a density sufficient for the analysis of individual cells. Well-developed myofibrillar structure was detected by titin and $\alpha$-actinin immunocytology (Fig. $2, E$ and $F$, respectively). Intermediate filament structure typical of cardiomyocytes was detected by desmin immunocytology (Fig. $2 G$ ). High power views of the MHC immunoreactivity revealed the presence of well-aligned myofibers in adjacent G418-selected cells (Fig. $2 H$ ). Dystrophin immunoreactivity was also detected in the selected cardiomyocyte cultures (Fig. $2 \mathrm{I}$ ); the dystrophin gene product is a well-established marker for both cardiac and skeletal muscle. The immunocytological analyses of the selected EA3 cultures are summarized in Table II. In each instance, cardiac tissue from normal fetal, normal adult, and dystrophic adult mice were included as controls.

Heterogeneous ANF immunoreactivity was observed in G418-selected EA3 cultures (Table II). It is well-established that ANF is expressed constitutively in the atrium during development. Although ANF is also expressed at high levels in the fetal ventricle, both transcription and protein accumulation at this site are dramatically downregulated at birth (24, see also Table II). Thus, the heterogeneous ANF expression observed in selected EA3 cultures suggests that these cells have differentiated into the adult phenotype, and that cells with attributes similar to either atrial or ventricular cardiomyocytes are present.

To further address this issue, Northern blots were performed with RNA prepared from adult atria, adult ventricles, undifferentiated EA3 cells, and G418-selected EA3 cardiomyocyte cultures. The blots were hybridized with oligonucleotide probes from the $3^{\prime}$ untranslated region of the MLC-2 $v$ and MLC-2a genes (Fig. 3). The hybridization signals observed with the atrial and ventricular RNA samples established the relative specificity of the probes. No signal was observed with RNA prepared from undifferentiated EA3 cultures. In contrast, both the MLC-2v and MLC-2a probes hybridized to RNA prepared from selected EA3 cultures, consistent with the notion that both atrial- and ventricular-like cardiomyocytes can be selected by this process. The relatively low MLC$2 \mathrm{v}$ and MLC-2a signal observed in the selected cardiomyocyte cultures as compared with the dissected tissue samples most likely reflects the fact that the cultures are a mixture of atrialand ventricular-like cardiomyocytes where the tissue samples were not. The potential contribution of conduction cells to the selected cultures would also impact on the relative MLC signals. In addition, the cultured cells were not under mechanical load, and as such MLC-2 transcription may not have been 


\begin{tabular}{|c|c|c|c|c|}
\hline \multirow[b]{2}{*}{ Marker* } & \multicolumn{4}{|c|}{ Cell type } \\
\hline & $\begin{array}{l}\text { Normal embryonic } \\
\text { cardiac muscle }\end{array}$ & $\begin{array}{l}\text { Normal adult } \\
\text { cardiac muscle }\end{array}$ & $\begin{array}{l}\text { Dystrophic adult } \\
\text { cardiac muscle }\end{array}$ & $\begin{array}{c}\text { G418-selected } \\
\text { cardiomyocytes }\end{array}$ \\
\hline $\begin{array}{l}\text { Myosin heavy chain } \\
\text { (MF20) }\end{array}$ & $+^{\ddagger}$ & + & + & + \\
\hline $\begin{array}{l}\text { Titin } \\
\quad \text { (9D10) }\end{array}$ & + & + & + & + \\
\hline $\begin{array}{l}\alpha \text {-Actinin } \\
\quad(\text { EA-53) }\end{array}$ & + & + & + & + \\
\hline $\begin{array}{l}\text { Desmin } \\
\text { (D3) }\end{array}$ & + & + & + & + \\
\hline $\begin{array}{l}\text { Nebulin } \\
\text { (NB2) }\end{array}$ & - & - & - & - \\
\hline $\begin{array}{l}\text { ANF } \\
\quad(\text { MA-APIII) }\end{array}$ & + & $\pm^{\S}$ & $\pm^{\S}$ & $\pm^{\S}$ \\
\hline $\begin{array}{l}\text { Dystrophin } \\
(6-10)\end{array}$ & \pm & + & - & + \\
\hline $\begin{array}{l}\text { Dystrophin } \\
\text { (DYS1) }\end{array}$ & \pm & + & - & + \\
\hline $\begin{array}{l}\text { Utrophin } \\
\text { (DRP2) }\end{array}$ & $\pm \|$ & - & + & $\pm \|$ \\
\hline
\end{tabular}

*EA-53 and NB2 were from Sigma Immunochemicals, DYS1 and DRP2 were from Novocastra Laboratories. Sources for the other antibodies are listed in the Methods and Acknowledgments section. ${ }^{*}+$ indicates strong signal; \pm indicates heterogenous signal; - indicates no signal. ${ }^{\S}$ ANF was detected in the atria but not ventricles of all adult histologic sections. Only a small percentage of the G418-selected cardiomyocytes exhibited ANF immunoreactivity. "Utrophin immunoreactivity was heterogeneous in normal embryonic cardiac muscle and in G418-selected cardiomyocytes.

maximally induced. Finally, the RNA input in the selected cardiomyocyte lanes was low compared with the tissue controls (compare the relative 18s rRNA signals).

The G418-selected cardiomyocytes exhibited spontaneous and rhythmic contractile activity, with adjacent cells beating

\section{MLC-2v}

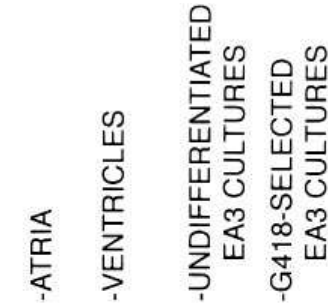

MLC-2a
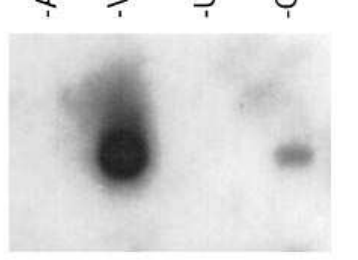

Figure 3. MLC-2a and MLC-2v are expressed in G418-selected EA3 cultures. RNA from adult atria, adult ventricles, undifferentiated EA3 cells, or G418selected EA3 cultures was hybridized to oligonucleotide probes from the MLC-2v (top) or MLC-2a (middle) genes. The integrity and quantity of the RNA samples was assessed by hybridization with an 18 s rRNA probe (bottom). synchronously. Cultures of selected, beating cardiomyocytes could be maintained for as long as 11 mo (Klug, M.G., unpublished observation). Ultrastructural analyses further established the differentiated status of EA3 cardiomyocyte cultures (Fig. 4). Normal adult sarcomeric structure was apparent in the selected cells. Intercalated discs composed of fascia adherens, desmosomes, and gap junctions were observed to connect adjacent cells (Fig. $4 \mathrm{~B}$ ). The laminar structure of the gap junctions was confirmed by examination at high magnification (Fig. 4 C). Myosin synthesis was observed to occur along free ribosomes in many of the selected cells (Fig. $4 D$ ). Collectively, these light and electron microscopic analyses indicated that G418 selection did not impact negatively upon cardiomyocyte differentiation.

Selected cardiomyocytes were tested for their ability to generate intracardiac grafts. G418-treated EA3 cultures were digested with trypsin, and $\sim 1 \times 10^{4}$ cells were delivered into the left ventricular free wall of dystrophic mdx recipient mice. Given that the selected cardiomyocytes expressed dystrophin (see Fig. 2 I and Table II) whereas the myocardium of the recipient mdx mice did not (see Table II, also reference 25), the fate of engrafted donor cells was readily monitored by antidystrophin immunohistology. Phase-contrast microscopic examination of cryosections of the recipient hearts revealed that engrafted regions frequently exhibited normal myocardial topography (Fig. $2 \mathrm{~J}$ ). Immunocytologic assays with antidystrophin antibody 6-10 revealed the presence of dystrophin-positive G418-selected cardiomyocytes (Fig. $2 \mathrm{~K}$ and $L$ ). Comparison of phase-contrast and antidystrophin images of the same field revealed the presence of myofibers in the engrafted G418-selected cardiomyocytes (Fig. 2, $J$ and $K$, respectively). 

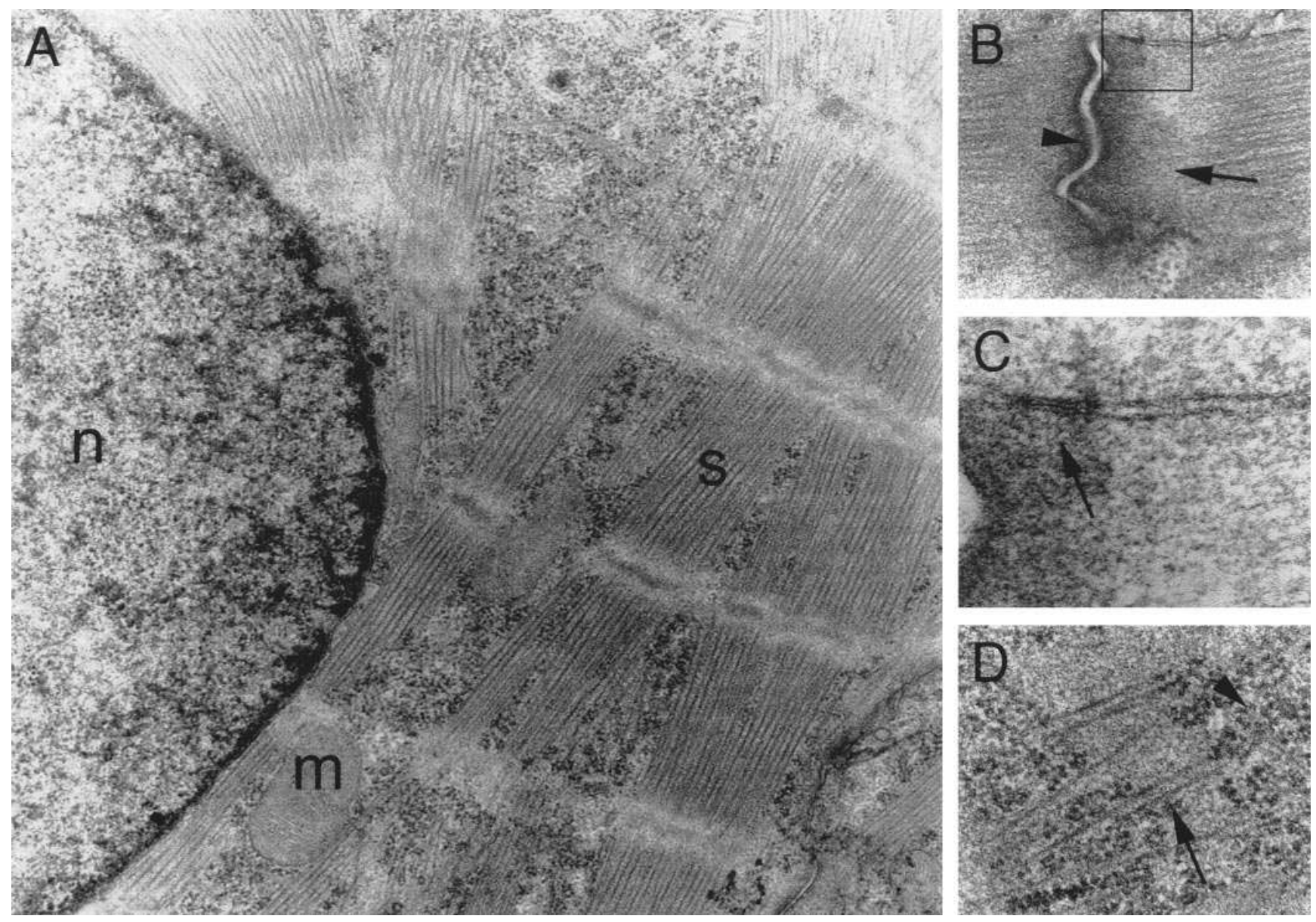

Figure 4. Ultrastructural analysis of selected EA3 cardiomyocytes. (A) Low-power transmission electron micrograph of a G418-selected cardiomyocyte: $n$, nucleus; $m$, mitochondria; $s$, sarcomere. $(B)$ High-power electron micrograph showing the presence of a desmosome (arrowhead), fascia adherens (arrow), and gap junctions (boxed), which are the ultrastructural components of intercalated discs. $(C)$ High-power view of the boxed region in $B$ demonstrating the laminar structure of the gap junctions. $(D)$ High-power electron micrograph demonstrating the presence of myosin synthesis (arrows) along free ribosomes (arrowheads).

6-10, a relatively high-affinity polyclonal antibody, recognizes the rod domain of dystrophin (amino acid residues 19913112). To confirm that this immunoreactivity was not artifactual (i.e., an "immunologic revertant," see reference 25), adjacent sections of the engrafted hearts were examined with an antidystrophin monoclonal antibody (DYS1) which recognizes a different epitope (amino acid residues 1181-1388). 83\% of the 6-10 positive grafts exhibited DYS1 immunoreactivity on adjacent sections (Table III). Importantly, no DYS1 immunoreactivity was observed in multiple sections of control mdx samples. Given the unequivocal nature of the DYS1 control experiment, the positive signal observed in these hearts strongly suggests that the G418-selected cardiomyocytes were engrafted successfully. Six of the eight animals analyzed developed grafts exhibiting both 6-10 and DYS1 immunoreactivity; a similar success rate was obtained with fetal cardiomyocyte grafts $(7,8)$.

Previous studies with a canine dystrophic model used utrophin immunohistology to provide a second, albeit negative, marker for engrafted cardiomyocytes (8). Although utrophinnegative, dystrophin-positive cardiomyocytes were detected in the engrafted hearts in this study, the heterogeneous pattern of utrophin expression observed in cultured EA3 cardiomyocytes precluded the use of this assay as second marker for engrafted cells. Therefore PCR amplification with primers specific for the MHC-neo ${ }^{r}$ fusion gene was used to confirm the presence of engrafted G418-selected cardiomyocytes. DNA was prepared

Table III. Summary of Dystrophin Immunoreactivity and PCR Analyses of mdx Hearts Engrafted with G418-selected Cardiomyocytes

\begin{tabular}{lccc}
\hline Mouse & $\begin{array}{c}\text { Dystrophin } \\
(6-10)\end{array}$ & $\begin{array}{c}\text { Dystrophin } \\
\text { (DYS1) }\end{array}$ & PCR \\
\hline 1 & $+*$ & + & - \\
2 & + & - & ND $^{\ddagger}$ \\
3 & - & - & ND \\
4 & + & + & + \\
5 & - & - & ND \\
6 & + & + & + \\
7 & + & + & + \\
8 & + & & + \\
\hline
\end{tabular}

* + indicates signal detected; - indicates no signal detected; ND indicates not determined. ${ }^{\ddagger}$ PCR analyses were performed only on those grafts which exhibited both 6-10 and DYS1 immunoreactivity. 
EA3 CELLS

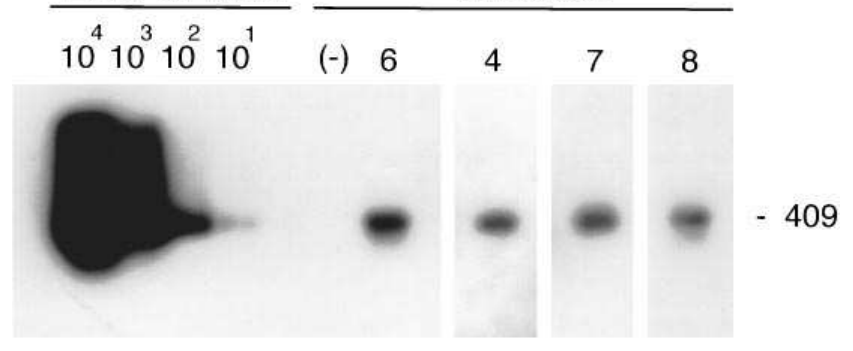

Figure 5. Southern blot analysis of PCR-amplified DNA prepared from mdx hearts engrafted with G418-selected ES-derived cardiomyocytes. DNA from $10^{4}, 10^{3}, 10^{2}$, or $10^{1}$ EA3 cells, from a nonengrafted heart section (-), or from engrafted heart sections exhibiting DYS1 immunoreactivity was amplified with primers specific for the MHCneo $^{r}$ transgene (mouse number refers to the designations presented in Table III). The resulting amplification products were separated on agarose gels, transferred to nylon membrane, and hybridized with a nick-translated probe corresponding to sequences of the transgene internal to the primers used for PCR amplification. The 409-bp MHC-neo ${ }^{r}$ transgene amplification product was readily detected in the samples prepared from DYS1-positive engrafted hearts, as well as in the positive control samples from EA3 cells. No signal was detected in the amplification products generated from nonengrafted heart sections.

from engrafted heart sections exhibiting DYS1 immunoreactivity, as well as from sections of nonengrafted control hearts. DNA from the parental EA3 cell line was used as a positive control. The DNA samples were subjected to PCR amplification, and the products were separated on agarose gels, transferred to nylon membranes, and hybridized with a nick-translated probe which encompassed sequences of the MHC-neor transgene internal to the oligonucleotide primers used for PCR amplification. Amplification of control EA3 DNA produced an appropriately sized band which hybridized to the transgene probe on the Southern blot (Fig. 5). An identical band was observed in amplification products of DNA prepared from DYS1-positive sections, but not in the amplification products of nonengrafted samples (Fig. 5). These results provided a second independent marker for the presence of engrafted G418-selected cardiomyocytes. The results of the graft analyses are summarized in Table III.

\section{Discussion}

This report describes a simple genetic modification which permitted the generation of essentially pure cultures of cardiomyocytes from differentiating ES cells. Cardiomyocytes expressing an MHC-neo ${ }^{\mathrm{r}}$ transgene survived G418 selection and remained stable in culture for extended periods of time. In contrast, noncardiomyocyte cell lineages derived from the parental ES cell did not express the fusion gene and consequently were eliminated by G418 selection. This procedure reproducibly yielded cardiomyocyte cultures which were $>99 \%$ pure, as based on sarcomeric myosin immunoreactivity in isolated cell preparations. Expression of the MHC-neor transgene did not impact negatively on cardiogenic differentiation, as evidenced by the spontaneous contractile activity of selected cells. This observation was supported by both immunofluorescence and ultrastructural analyses, which documented the presence of well-developed myofibers in the selected cells. Intercalated discs were observed to couple juxtaposed selected cardiomyocytes, consistent with the observation that adjacent cells exhibited synchronous contractile activity. Collectively these data indicate that the selection process gives rise to highly differentiated cells. Furthermore, the selected cardiomyocytes were able to form stable intracardiac grafts, with the engrafted cells aligned with host cardiomyocytes.

The differentiated status of ES-derived cardiomyocytes is well established. Molecular, cytological, electrophysiological, and contractile analyses have demonstrated that ES-derived cardiomyocytes exhibit many of the physical and molecular attributes observed in typical cardiomyocytes (9-18). Although the analyses of selected EA3-derived cardiomyocytes presented here were of a more limited scope, all of the markers examined were consistent with a highly differentiated cardiomyocyte preparation. A priori, one would anticipate that the entire spectrum of differentiated characteristics seen in naive ES-derived cardiomyocyte preparations would also be present in the EA3-selected cells. Refinement of the selection procedure (i.e., selection of atrial versus ventricular cardiomyocytes, or selection of conduction system versus working cardiomyocytes) should be possible simply by choosing an appropriately cell type-restricted promoter to target aminoglycoside phosphotransferase expression. Combinatorial approaches using multiple promoters and selectable markers also can be envisioned. The observation that cells with attributes similar to atrial or ventricular cardiomyocytes were present in G418selected cultures bodes well for the feasibility of isolating specific cardiomyocyte subtypes.

The approach presented here can generate as many as $10^{6}$ ES-derived cardiomyocytes with an initial input of $10^{6}$ stem cells. However, the inherent variability of cardiogenic induction in differentiating ES cultures can have a significant impact on the ultimate yield in a given preparation. Preliminary studies have indicated that many factors can influence the cardiomyocyte yield. These factors include serum content, length of growth in suspension culture, and initial embryoid body plating density. Thus, while the yield of G418-selected cardiomyocytes currently is somewhat variable, it is likely that optimization and standardization of the induction, culture, and selection protocols will result in improved and more reproducible preparations.

Cellular engraftment is emerging as a viable approach to augment myocyte number in adult hearts. To date several myocyte preparations have been grafted successfully into the adult myocardium. These include AT-1 tumor cardiomyocytes $(1,2)$, both naive and genetically engineered myoblasts (3-6), and fetal cardiomyocytes $(7,8)$. The limited studies reported to date suggest that fetal cardiomyocyte engraftment may hold the greatest potential to effect myocardial repair, as the engrafted donor cells were observed to form nascent intercalated discs with host cardiomyocytes $(7,8)$. While it remains to be established if any form of cellular engraftment can effect myocardial repair (see reference 26 for a critical review), it is virtually certain that human fetal donor cardiomyocytes cannot be obtained in sufficient numbers for use in a clinical setting. As such, identification of an alternative source of donor cardiomyocytes would constitute an important advancement. The observation that selected EA3 cardiomyocytes form stable intracardiac grafts raises the possibility that ES-derived cells may constitute a viable alternative source of donor cells. Im- 
portantly, the frequency of successful engraftment with EA3 cardiomyocytes was similar to that observed with fetal murine cardiomyocytes $(7,8)$. Engrafted myofiber-containing donor cells were observed to be aligned and tightly juxtaposed with host cardiomyocytes. The generation of stable intracardiac grafts in the absence of teratoma formation further attests to both the cardiomyocyte purity and cell cycle withdrawal attained with the selected cultures. Collectively these observations suggest that ES-derived cardiomyocyte preparations may be a useful surrogate for fetal cardiomyocytes in cardiac engraftment procedures.

The EA3 cells also may prove to be a useful cell culture resource. Given the limited proliferative capacity of naive mammalian cardiomyocytes (27), most in vitro experiments have used primary cultures of fetal or neonatal cells. Although the utility of such cell preparations is well established, the cultures are cumbersome to generate and frequently can be overrun by fibroblasts. Efforts to generate myocardial cell lines have focused largely on the targeted expression of oncogenes, either in cultured cardiomyocytes or in the myocardium of transgenic animals (for reviews see references 28 and 29). Although numerous cell culture resources have been generated in this manner, to date no established cell lines exhibiting contractile activity after repeated passage have been reported. While the EA3 ES cells can be cultured and passaged in the undifferentiated state, it should be stressed that the proliferative capacity of the nascent cardiomyocytes is limited. Indeed, previous analyses of nonselected cultures have indicated that ESderived cardiomyocytes withdraw from the cell cycle in a temporal pattern roughly similar to that observed for cardiomyocytes during normal murine development (18). Moreover, ESderived cardiomyocyte cell cycle withdrawal was accompanied by a shift toward multinucleation, a hallmark of cardiomyocyte cell cycle withdrawal in vivo. While the selection procedure described here does not provide a source of continuously proliferating cardiomyocytes, the ability to propagate the parental EA3 cell provides a continuously renewable ex vivo source of pure cardiomyocyte cultures. Importantly, selected cardiomyocytes with spontaneous contractile activity can be cultured for extended periods of time without encroachment by other cell lineages.

The selection procedure described here should be applicable to all ES-derived cell lineages, provided that suitable cell type-specific promoters are available. Given that numerous cell lineages of ecto-, endo-, and mesodermal origin are represented in differentiating ES cultures (9), it is likely that ESderived cellular transplantation strategies can be extended to other organ systems. To date, ES lines capable of cardiogenic differentiation have been generated in a number of species, including mouse, rat, rabbit, mink, pig, and most recently primates (30). If cardiomyocyte engraftment proves to be of therapeutic value, the generation of cardiogenic human ES cell lines would preclude the requirement for either human fetal or xenotrophic tissue. The existence of pluripotent human embryonic carcinoma cell lines is encouraging with regards to the prospects of generating cardiogenic human ES cells.

\section{Acknowledgments}

We thank H. Wang and D. Field for excellent technical assistance, Rick Mortensen (Harvard Medical School, Cambridge, MA) for the
PGK-hygromycin transgene, J. Robbins (University of Cincinnati School of Medicine, Cincinnati, OH) for the mouse $\alpha$-cardiac MHC promoter, Austin G. Smith (University of Edinburgh, Edinburgh, Scotland) for the LIF expression vector, Chris Glembotski (San Diego State University, San Diego, CA) for MA-APIII antibody, Jeff Robbins (CHRF, Cincinnati, OH) for the MLC-2a and MLC-2v oligonucleotides, and Tim Byers (Indiana University) for 6-10 antibody. MF20, D3, and 9D10 were obtained from the Developmental Studies Hybridoma Bank maintained by the Department of Pharmacology and Molecular Sciences, Johns Hopkins University School of Medicine (Baltimore, MD) and the Department of Biological Sciences, University of Iowa (Iowa City, IA), under contract N01-HD-2-3144 from the National Institute of Child Health and Human Development.

This work was supported by grants HL-53505 and HL-52323 from the National Heart, Lung and Blood Institute. This work was done during the tenure of an Established Investigatorship from the American Heart Association (L.J. Field). M.G. Klug was supported by a predoctoral fellowship from the American Heart Association, Indiana Affiliate.

\section{References}

1. Koh, G.Y., M.H. Soonpaa, M.G. Klug, and L.J. Field. 1993. Long-term survival of AT-1 cardiomyocyte grafts in syngeneic myocardium. Am. J. Physiol. 264:H1727-H1733.

2. Delcarpio, J.B., and W.C. Claycomb. 1995. Cardiomyocyte transfer into the mammalian heart: cell-to-cell interactions in vitro and in vivo. Ann. NY Acad. Sci. 752:267-285.

3. Koh, G.Y., M.G. Klug, M.H. Soonpaa, and L.J. Field. 1993. Differentiation and long-term survival of $\mathrm{C} 2 \mathrm{C} 12$ myoblast grafts in heart. J. Clin. Invest. 92:1548-1554

4. Chiu, R.C., A. Zibaitis, and R.L. Kao. 1995. Cellular myoplasty: myocardial regeneration with satellite cell implantation. Ann. Thorac. Surg. 60:12-18.

5. Robinson, S.W., P.C. Cho, M.A. Acker, and P.D. Kessler. 1994. Arterial delivery of skeletal myoblasts to the heart. J. Cell. Biochem. 18D:531a (Abstr.)

6. Koh, G.Y., S.J. Kim, M.G. Klug, K. Park, M.H. Soonpaa, and L.J. Field. 1995. Targeted expression of TGF- $\beta 1$ and neovascularization in hearts bearing intracardiac grafts. J. Clin. Invest. 95:114-121.

7. Soonpaa, M.H., G.Y. Koh, M.G. Klug, and L.J. Field. 1994. Formation of nascent intercalated discs between grafted fetal cardiomyocytes and host myocardium. Science (Wash. DC). 264:98-101.

8. Koh, G.Y., M.H. Soonpaa, M.G. Klug, H.P. Pride, D.P. Zipes, B.J. Cooper, and L.J. Field, 1995. Stable fetal cardiomyocyte grafts in the hearts of dystrophic mice and dogs. J. Clin. Invest. 96:2034-2042.

9. Doetschman, T.C., H. Eistetter, M. Katz, W. Schmidt, and R. Kemler. 1985. The in vitro development of blastocyst-derived embryonic stem cell lines: formation of visceral yolk sac, blood islands and myocardium. J. Embryol. Exp. Morphol. 87:27-45.

10. Sanchez, A., W.K. Jones, J. Gulick, T. Doetschman, and J. Robbins. 1991. Myosin heavy chain gene expression in mouse embryoid bodies. An in vitro developmental study. J. Biol. Chem. 266:22419-22426.

11. Muthuchamy, M., L. Pajak, L. Howles, T. Doetschman, and D.F. Wieczorek. 1993. Developmental analysis of tropomyosin gene expression in embryonic stem cells and mouse embryos. Mol. Cell. Biol. 13:3311-3323.

12. Miller-Hance, W.C., M. LaCorbiere, S.J. Fuller, S.M. Evans, G. Lyons, C. Schmidt, J. Robbins, and K.R. Chien. 1993. In vitro chamber specification during embryonic stem cell cardiogenesis. Expression of the ventricular myosin light chain-2 gene is independent of heart tube formation. J. Biol. Chem. 268: 25244-25252.

13. Ganim, J.R., W. Luo, S. Ponniah, I. Grupp, H.W. Kim, D.G. Ferguson, V. Kadambi, J.C. Neumann, T. Doetschman, and E.G. Kranias. 1992. Mouse phospholamban gene expression during development in vivo and in vitro. Circ. Res. 71:1021-1030.

14. Boer, P.H. 1994. Activation of the gene for type-b natriuretic factor in mouse stem cell cultures induced for cardiac myogenesis. Biochem. Biophys. Res. Commun. 199:954-961.

15. Metzger, J.M., W.-I. Lin, and L.C. Samuelson. 1994. Transition in cardiac contractile sensitivity to calcium during the in vitro differentiation of mouse embryonic stem cells. J. Cell Biol. 126:701-711.

16. Maltsev, V.A., J. Rohwedel, J. Hescheler, and A.M. Wobus. 1993. Embryonic stem cells differentiate in vitro into cardiomyocytes representing sinusnodal, atrial and ventricular cell types. Mech. Dev. 44:41-50.

17. Wobus, A.M., G. Wallukat, and J. Hescheler, 1991. Pluripotent mouse embryonic stem cells are able to differentiate into cardiomyocytes expressing chronotropic responses to adrenergic and cholinergic agents and $\mathrm{Ca}++$ channel blockers. Differentiation. 48:173-182. 
18. Klug, M.G., M.H. Soonpaa, and L.J. Field. 1995. DNA synthesis and multinucleation in embryonic stem cell-derived cardiomyocytes. Am. J. Physiol. 269:H1913-H1921.

19. Gulick, J., A. Subramaniam, J. Neumann, and J. Robbins. 1991. Isolation and characterization of the mouse cardiac myosin heavy chain genes. $J$. Biol. Chem. 266:9180-9185.

20. Mortensen, R.M., D.A. Conner, S. Chao, L.A. Geisterfer, and J.G. Seidman. 1992. Production of homozygous mutant ES cells with a single targeting construct. Mol. Cell. Biol. 12:2391-2395.

21. Smith, A.G. 1991. Culture and differentiation of embryonic stem cells. J. Tissue Culture Methods. 13:89-94.

22. Sambrook, J., E.F. Fritsch, and T. Maniatis. 1989. Molecular Cloning: A Laboratory Manual. Second edition. Cold Spring Harbor Press, Cold Spring Harbor, NY.

23. Steinhelper, M.E., K. Cochrane, and L.J. Field. 1990. Hypotension in transgenic mice expressing atrial natriuretic factor fusion genes. Hypertension (Dallas). 16:301-307.

24. Zeller, R., D. Bloch, B.S. Williams, R.J. Arceci, and C.E. Seidman. 1987. Localized expression of the atrial natriuretic factor gene during cardiac em- bryogenesis. Genes \& Dev. 1:693-698.

25. Danko, I., V. Chapman, and J.A. Wolff. 1992. The frequency of revertants in mdx mouse genetic models for Duchenne muscular dystrophy. Pediatr. Res. 32:128-131

26. Soonpaa, M.H., M.G. Klug, H. Nakajima, H. Nakajima, and L.J. Field. 1996. Potential approaches for cell-mediated myocardial repair. In Unstable Coronary Artery Disease: Pathophysiology, Diagnosis and Treatment. R.W. Wilensky, editor. Kluwer Academic Press, New York. In press.

27. Rumyantsev, P.P. 1991. Reproduction of cardiac myocytes developing in vivo and its relation to processes of differentiation. In Growth and Hyperplasia of Cardiac Muscle Cells. B.M. Carlson, editor. Harwood Academic Press, New York. 70-157.

28. Field, L.J. 1993. Transgenic mice in cardiovascular research. Ann. Rev. Physiol. 55:97-114.

29. Daud, A.I., and L.J. Field. 1993. Interaction between host proteins and SV40 large T-antigen in transformed cardiomyocytes. Heart Failure. 9:183-198.

30. Thomson, J.A., J. Kalishman, T.G. Golus, M. Durning, C.P. Harris, R.A. Becker, and J.P. Hearn. 1995. Isolation of a primate embryonic stem cell. Proc. Natl. Acad. Sci. USA. 92:7844-7848. 